\title{
Un Programa de Aprendizaje Autorregulado para Personas de Altas Capacidades Mediante el Uso de Herramientas Telemáticas
}

\section{Carmen M. Hernández Jorge ${ }^{1}$ África Borges del Rosal ${ }^{2}$}

${ }^{1}$ Dpto. de Psicología Evolutiva y de la Educación, Universidad de La Laguna 2 Dpto. de Psicobiología y Metodología de las Ciencias del Comportamiento, Universidad de La Laguna

España 


\section{Resumen}

Poseer altas capacidades intelectuales no resulta una garantía de éxito académico. De hecho, se suele informar en la literatura especializada de problemas de retraso escolar, así como de hiperactividad y problemas atencionales en el alumnado de altas capacidades. Ello hace que sea relevante diseñar programas que ayuden a la obtención de éxito académico. Se presenta aquí un programa virtual, Navegando en el aprendizaje, basado en estrategias de aprendizaje y aprendizaje autorregulado, dirigido a alumnado superdotado, con la finalidad de prevenir problemas académicos, contribuyendo a mejorar sus destrezas para el estudio y el rendimiento.

Palabras clave: Superdotación, estrategias de aprendizaje, contextos hipermedia 
Introducción

Una paradoja con la que se enfrenta el estudioso de la superdotación es que alumnos o alumnas con una alta capacidad intelectual pueden presentar problemas de aprendizaje. Los factores explicativos de este fenómeno no se han aislado convenientemente. Así, mientras Castor (1997) encuentra que es el autoconcepto académico lo que diferencia entre superdotados con buen y mal rendimiento, Lee-Corbin y Denicolo (1998) y Reis y McCoach (2000) concluyen que concurren múltiples factores, siendo raros los casos en que se pueda aislar una sola variable.

En ocasiones, lo que provoca desajustes en el rendimiento académico es algo tan simple como el aburrimiento, que hace que el niño o la niña superdotados, cansados de tareas repetitivas, deje de prestar atención y pierda interés por las tareas académicas (Bragget, Ashman y Noble, 1983; Kunkel, Chapa, Petterson y Walling, 1992; Shultz, 2003). Otras veces, la relación con alguno de los profesores es claramente inadecuada, cuestión que además de venir recogida en la literatura (véase, por ejemplo, Reis y Colbert, 2004) hemos tenido ocasión de contrastarlo en las experiencias de los niños y niñas participantes en nuestro programa.

Lo que sí parece claro, como señalan Zentall, Hall y Grskovic (2001), es que la superdotación no protege de tener problemas de atención y de bajo rendimiento en la escuela.

Entre los problemas más comúnmente descritos en la literatura se encuentran los déficits de atención e hiperactividad. Si bien existe la posibilidad de que haya dificultades con el diagnóstico diferencial (Kaufmann, Kalbfleisch y Castellanos; 2000; Hartnett, Nelson y Rinn, 2004), hay muchas ocasiones donde se conjugan superdotación y déficits atencionales y de hiperactividad (véase, por ejemplo, el trabajo de Zentall, Hall y Grskovic, 2001)

El problema de un bajo rendimiento no se limita al mundo académico, siendo esto en sí ya grave al truncar carreras, sino que tiene consecuencias a la larga, pudiendo padecer años de dificultades personales, que puede llegar incluso a límites como consumo de drogas e intentos de suicidio (Flint, 2003). 
No obstante, cabe la posibilidad de establecer soluciones para intentar solventar los problemas de rendimiento. Así, Reis y Colbert (2004) presentan labores de asesoría para aquellos estudiantes con altas capacidades y problemas académicos, o bien se realizan programas específicos para estas personas (Weinfeld, Barnes-Robinson, Jeweler y Shevitz, 2002).

La perspectiva de las dificultades mencionadas nos ha llevado a elaborar un programa, con carácter preventivo, que aúna estrategias de aprendizaje con aprendizaje autorregulado, impartido de forma virtual. Pasamos a continuación a plantear las justificaciones del modelo de programa realizado.

\section{Estrategias de aprendizaje}

Existen diferentes factores en el alumnado que influyen en el rendimiento académico. Según la teoría de la potencia cognitiva de Hernández y García (1991), completada por García (1998), existen diferentes aspectos que se relacionan con el rendimiento académico (véase gráfica número 1). Los autores señalan que las variables más alejadas de la base de la pirámide son los aspectos que menos se relacionan con el rendimiento académico, tales como el factor ' $G$ ' de la inteligencia o las aptitudes mentales primarias (atención, memoria, etc.), mientras lo más cercano a la base de la pirámide son los aspectos más relacionados con el rendimiento, esto es, los contenidos escolares. En un nivel intermedio se sitúan las estrategias de aprendizaje, que son las habilidades que utilizan los estudiantes para aprender dichos contenidos curriculares.

\section{Gráfica 1: Pirámide de Potencia Cognitiva (Hernández y García, 1991; García, 1998)}

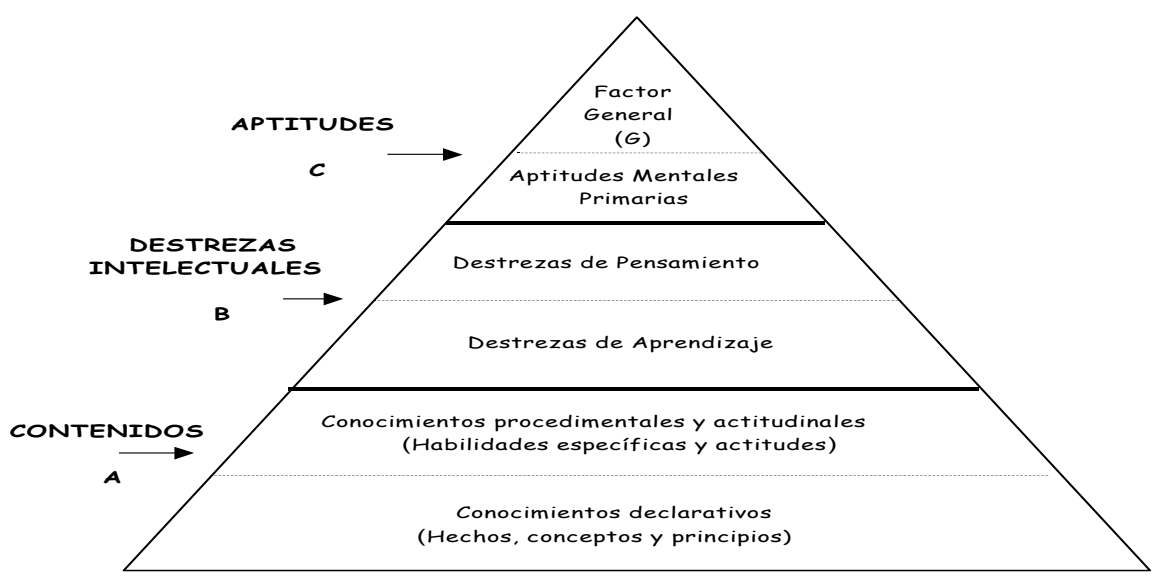


En la intervención en los tres factores comentados (factor g, estrategias de aprendizaje y contenidos) los autores citados señalan que la adquisición de contenidos académicos es lo que más se relaciona con el rendimiento. No obstante, este tipo de aprendizaje, muy puntual y ligado al texto, resulta poco generalizable para acceder a otros contenidos, mientras que la intervención que se realiza en las estrategias de aprendizaje tiene mayor poder de generalización a distintos contenidos, por lo que resulta más eficaz y duradera, con lo que "el tramo de las estrategias de pensamiento y de las estrategias de aprendizaje es la parte más óptima para la intervención” (García, Torbay, Rodríguez, Martín, Rodríguez y HernándezJorge, 2003, p. 88).

Por otro lado, existen una serie de consideraciones de la variable rendimiento académico, ya que ésta tiene que ver con el tipo de evaluación que realiza el docente y el tipo de tarea que se solicita al alumnado. De manera que cuando se solicitan tareas evaluativas que incluyen estrategias de aprendizaje, tales como relacionar la información, estructurarla o elaborarla, la relación entre estrategias de aprendizaje y rendimiento aumentaría (Torbay, 2002), con lo que parece fundamentarse la importancia de la intervención y mejora de las estrategias de aprendizaje para incrementar el rendimiento académico de los estudiantes.

Algunos estudios señalan, incluso, que las personas creativas utilizan diferentes estrategias de aprendizaje. Así, estas personas utilizan estrategias para estudiar más elaborativas (relación de la información, realización de autopreguntas, etc), de comprensión elaborada (llevar la teoría a aspectos experienciales propios, plantear con otras palabras lo que está en el texto, etc.), de estructuración de la información o esencialización de la misma (Torbay, Martín, García y Rodríguez, 2001a y 2001b).

\section{Aprendizaje autorregulado}

Educar implica potenciar el desarrollo de la persona en todas sus capacidades. Por ello los fines educativos deben abarcar "la realidad integral del ser humano" (Hernández, 1991, p. 232). De ahí que los fines intelectivos de la educación, más relacionados con el aprendizaje y el rendimiento, tengan que ver con la cantidad de información que se ofrece (objetivos informativos) y con el desarrollo de habilidades cognitivas que permitan el manejo de la información proporcionada (objetivos formativos). Estos objetivos formativos se gradúan en 
un continuo de reproductividad-productividad de la información, el cual alude a las habilidades cognitivas que se requieren para manejar la información. De esta forma, en el extremo del polo reproductivo se sitúan habilidades que 'reproducen' la información (memoria, comprensión informativa), mientras que en el polo productivo se sitúan habilidades cognitivas que requieren un manejo más complejo de la información (asociación, razonamiento, análisis, aplicabilidad de contenidos, toma de decisiones, etc.) Este tipo de habilidades cognitivas responderían a una enseñanza relacionada con la corriente constructivista, en cuanto que toma como referencia objetivos que intentan desarrollar las potencialidades intelectivas del individuo para producir o crear información.

Por tanto, parece especialmente relevante ayudar a desarrollar en los estudiantes aprendizaje autorregulado, el cual se describe como el tipo de aprendizaje basado en el uso de estrategias metacognitivas a la hora de afrontar tareas de estudio, el uso de estrategias exitosas y acordes con las demandas de la tarea y el componente de motivación intrínseca por aprender (Zimmerman, 1990; Winne y Perry, 2000; De la Fuente y Martínez, 2000).

Las estrategias metacognitivas suponen el conocimiento de: las características de la tarea, de las propias habilidades de aprendizaje, del proceso cognitivo que demanda la tarea y de las estrategias que deben utilizarse (De la Fuente y Martínez, 2000). Como señalan Perry, Norbody y Vandekamp (2003), supone tomar conciencia de las propias fortalezas y debilidades, así como de las estrategias que deben utilizarse para afrontar las demandas de la tarea. El uso de estrategias exitosas implica tener un repertorio importante de habilidades de aprendizaje, de resolución de problemas y conocer de antemano qué demanda la tarea y que estrategia aplicar en cada caso (Zimmerman y Bandura, 1994). Además de estos aspectos intervienen las estrategias de control del estudio: planificación previa del proceso, regulación durante el mismo y evaluación final de las estrategias utilizadas (De la Fuente y Martínez, 2000). En definitiva, todas ellas pertenecen a habilidades propias de los metacomponentes de la teoría triárquica de la inteligencia propuesta por Stenberg (1988). Finalmente, la motivación intrínseca en la actividad implica el valor que le pone el estudiante a su progreso personal, la disposición positiva para afrontar desafíos que exigen desarrollar nuevas habilidades y la consideración constructiva del error como oportunidad para aprender (Pintrich y Schunk, 2002). 
En investigación concreta en esta temática, se ha tratado de promover aprendizaje autorregulado en las escuelas en diversos contenidos curriculares. Un ejemplo de ello es el trabajo de Perry, Norbody y Vandekamp (2003), en el que se estudia el entrenamiento en aprendizaje autorregulado en estudiantes de escuela primaria en la lectura y escritura. Estos autores señalan que mantener un ambiente rico en el aula (diversidad de actividades, debates, materiales, etc.) favorece que el alumnado genere habilidades y actitudes positivas hacia el aprendizaje autorregulado en esta materia concreta.

\section{Aprendizaje autorregulado y superdotación}

Las características que suelen relacionarse con superdotación parecen estar vinculadas a las destrezas que requiere el aprendizaje autorregulado (Zimmerman y Martínez-Pons, 1990). De aquí que se haya intentado comprobar si hay diferencias en autorregulación entre individuos con y sin altas capacidades intelectuales.

Parece haber evidencia de que las personas con altas capacidades utilizan más estrategias de autorregulación en el aprendizaje que las personas que no poseen estas altas capacidades, al mismo tiempo que tienen un mayor sentimiento de autoeficacia en la tarea (Zimmerman y Martínez-Pons, 1990), aspecto éste muy relacionado con la motivación intrínseca en la tarea y el aprendizaje (Ryan y Decy, 2000). De hecho, el sentimiento de competencia de los estudiantes en sus habilidades, las expectativas de éxito en la tarea, el valor que le otorga el estudiante a la actividad y su decisión de realizarla son predictoras del rendimiento académico y de la persistencia en la tarea (Wigfield y Eccles, 2000).

Profundizando más en las diferencias, otra línea de investigación se plantea si los estudiantes de altas capacidades poseen un repertorio de estrategias metacognitivas más amplias frente a las personas de capacidades 'normales'. Los resultados avalan este aserto, ya que los estudiantes de altas capacidades utilizan más estrategias de aprendizaje autorregulado que los estudiantes que no poseen estas características, y orientan más claramente sus metas al aprendizaje que las personas que no poseen alta capacidad (Zimmerman y Martínez-Pons, 1990; Jessie, Moore y Atputhasamy, 2003). Más allá de los aspectos diferenciales, una línea amplia de trabajos ahondan en la importancia de mejorar las estrategias cognitivas y metacognitivas que se sitúan a la base del aprendizaje autorregulado en estudiantes de altas 
capacidades, para optimizar el desarrollo de este potencial que poseen las personas superdotadas (Hacker, Dunlonsky y Graesser, 1998; Coltman, Devinney, Latukefu y Midgley, 2002; Zimmerman, 1995; Zimmerman y Schunk, 2001)

La comparación en áreas curriculares la ofrecen Neber y Schommer-Aikins (2002), quienes plantearon un trabajo comparando estudiantes de primaria con altas capacidades y estudiantes de secundaria sin esta característica. Los resultados de este estudio manifiestan que las experiencias de investigación en ciencias que se desarrolla en las aulas, incrementa la motivación del alumnado en el uso de estrategias de aprendizaje autorregulado. Con lo cual, los aspectos de aprendizaje basado en la resolución de problemas, la acción reflexionada y el aprendizaje por descubrimiento, en general, capacitan y fortalecen a los estudiantes con altas capacidades cuando trabajan el área de ciencias.

No puede perderse de vista que el aprendizaje está modulado por los aspectos afectivos de los estudiantes, los cuales favorecen o dificultan este proceso. Incluso ha sido comprobado a nivel multicultural, donde el autoconcepto de los estudiantes afecta significativamente a su rendimiento (Herrera, Ramírez, Roa, y Herrera, 2004). Así, estar pendiente de las comparaciones sociales en el grupo de clase (el mejor, el peor), de los refuerzos que ofrece el docente, mantener una actitud de evitación de fracaso, poseer un autoconcepto negativo sobre su propia 'valía' en el estudio, etc, interfieren en el desarrollo del estudiante y en el uso del aprendizaje autorregulado (Paris y Newman, 1990; Covington, 1992; Pintrich y Schunk, 2002). Se evidencia además que las personas que utilizan el aprendizaje autorregulado también poseen un alto sentimiento de autoeficacia y valoran positivamente el esfuerzo en el aprendizaje (Zimmerman y Martínez-Pons, 1990). Por otra parte, los estudiantes universitarios que utilizan habilidades de aprendizaje autorregulado se diferencian de los que no lo utilizan tanto en variables de tipo cognitivo (estrategias cognitivas, metacognitivas y motivacionales), como en variables afectivo-motivacionales (valor de la tarea, creencias de control, autoeficacia, ansiedad, metas académicas). Por tanto, existen estrechas relaciones entre los componentes cognitivo y afectivo-motivacional en el aprendizaje en general y también, por ende, en el aprendizaje autorregulado (González, Valle, Suárez y Fernández, 2000). Es decir, para el aprendizaje significativo y autorregulado se precisa voluntad y habilidad (McCombs y Marzano, 1990). Por lo tanto, un entrenamiento que introduzca elementos de aprendizaje autorregulado, no sólo tendrá efectos en la actividad 
académica del educando, sino que repercutirá, probablemente, en áreas afectivas y emocionales.

\section{Aprendizaje en los contextos hipermedia}

Los contextos hipermedia han 'revolucionado' la enseñanza y el aprendizaje, puesto que ofrecen un modelo más centrado en el alumnado y su proceso de aprender.

Diversos autores han señalado las ventajas que ofrece la teleformación, entre las que destacan: a) una clara independencia espacio-temporal, de manera que estudiantes, docentes $\mathrm{y}$ contenido no tienen que estar en un mismo lugar en un mismo tiempo, b) una mayor flexibilidad y posibilidad de adaptar el proceso de aprendizaje al alumnado, porque éste tiene mayor control sobre su aprendizaje (posibilidades de afianzarlo, horario, tiempo de acceso, etc.), lo cual puede favorecer el aprendizaje autónomo, ayudar a asumir mayor protagonismo o responsabilidad en él, etc., c) una mayor posibilidad de acceso a diferentes fuentes de información, porque se accede mediante diferentes vías a informaciones diversa, por diversos canales, vídeo, enlaces, etc., d) una mayor posibilidad de comunicación, ya que se da mayor interacción docente-alumnado y alumnado entre sí usando diversas herramientas telemáticas (correo electrónico, foros, chat, etc.), lo que puede favorecer que el estudiantado comparta tanto con el profesorado como con otros estudiantes, sus conocimientos, inquietudes, opiniones o sugerencias, e) una mayor posibilidad de personalización en el proceso de enseñanza, ya que el tutor/a, mediante las herramientas telemáticas, puede personalizar aún más su enseñanza, apoyo u orientación al alumnado, e) mayor probabilidad de reducir costes a largo plazo, porque llega a mayor número de estudiantes, se reduce el gasto de traslados, puede disponerse de mayor número de especialistas, etc. (Adell, 1997; Alcantud, 1999; Pérez, Rubio y Rubio, 1999; Rubio, 2000)

Todas estas ventajas se han incorporado a cursos on-line que tratan de mejorar las estrategias de aprendizaje del alumnado y el aprendizaje autorregulado específicamente. En ellos se introducen estrategias instruccionales que favorecen el aprendizaje significativo, basado en la acción y partiendo de solución de problemas para generar habilidades de pensamiento en los estudiantes de altas capacidades y estudiantes sin esta característica (Lee, 2001), demostrando que el entrenamiento mediante herramientas telemáticas en aprendizaje 
autorregulado facilita el cambio de 'modelos mentales' del alumnado para aprender mediante la red cuando se comparan con grupos de control (Azevedo y Cromley, 2004).

Como afirman Rogers y Shawn (2004) la innovación basada en las herramientas telemáticas ofrece a los estudiantes un ilimitado acceso a los recursos, y cuando los docentes generan estas posibilidades favorecen que los estudiantes desarrollen habilidades de pensamiento y estudio más sofisticados, tales como las que exige el aprendizaje autorregulado.

Existen diferentes estudios que nos orientan a pensar la importancia de usar los contextos hipermedia en la formación de las personas con altas capacidades. Algunos de ellos señalan que fomentan la creatividad y las estrategias de aprendizaje en este tipo de estudiantes (Bastani, 2004). También señalan que es importante el uso de las herramientas telemáticas para quienes quieran formar y promocionar a las personas con superdotación (Bertschi, 2004), ayudándoles a adquirir mayor conocimiento, habilidades de investigación y de pensamiento, incluso en edades tempranas (Burke y Tolan, 2004).

Tal como hemos señalado anteriormente la posibilidad de bajo rendimiento de los superdotados y la importancia de entrenar al alumnado en estrategias de aprendizaje que permitan su generalización a diferentes contenidos y que pueda generar un mejor rendimiento, nos ha llevado a desarrollar un programa de estrategias de aprendizaje para personas superdotadas. Por otra parte, las ventajas de los contextos hipermedia y la posibilidad de que ayuden a generar habilidades de pensamiento en el estudiantado, que le ofrezcan recursos diferentes y que estimulen su motivación por aprender, es lo que nos ha llevado a utilizar este medio para enseñar a aprender a las personas con superdotación, generando el programa Navegando en el aprendizaje.

\section{El programa navegando en el aprendizaje}

Todas las evidencias reseñadas anteriormente nos han llevado a generar un curso online dirigido a niños y niñas con altas capacidades desde una perspectiva extraescolar y con una base de aprendizaje basado en la acción, y en la resolución de problemas, a la vez que favorezca el aprendizaje autorregulado. Este curso se ubica en el Programa Integral Para Altas 
Capacidades (PIPAC), el cual integra, también formación a padres y madres y el desarrollo de los aspectos afectivos de los niños y niñas.

El programa está compuesto por siete unidades didácticas: recogida de información, estrategias de apoyo, estrategias de atención, estrategias para la elaboración y organización de la información, estrategias de memorización, la Integración personal de los conocimientos comprendidos y difusión de los conocimientos. Se accede a través de la página web del proyecto de investigación (http://gtisd.net), $y$, tras introducir clave de usuario, se llega a un menú gráfico (véase gráfica número 2), que representa las diversas unidades: una ventana para la recogida de información, un pupitre para las estrategias de apoyo, un búho para la atención, un archivador para las estrategias de elaboración y organización de la información, un ordenador para la memoria, un niño para las estrategias de personalización y una ventana para la difusión de conocimientos. Para la elaboración de los contenidos hemos acudido a Bernardo (2004) y a Cremades (2001)

\section{Gráfica 2: Menú de entrada al programa}

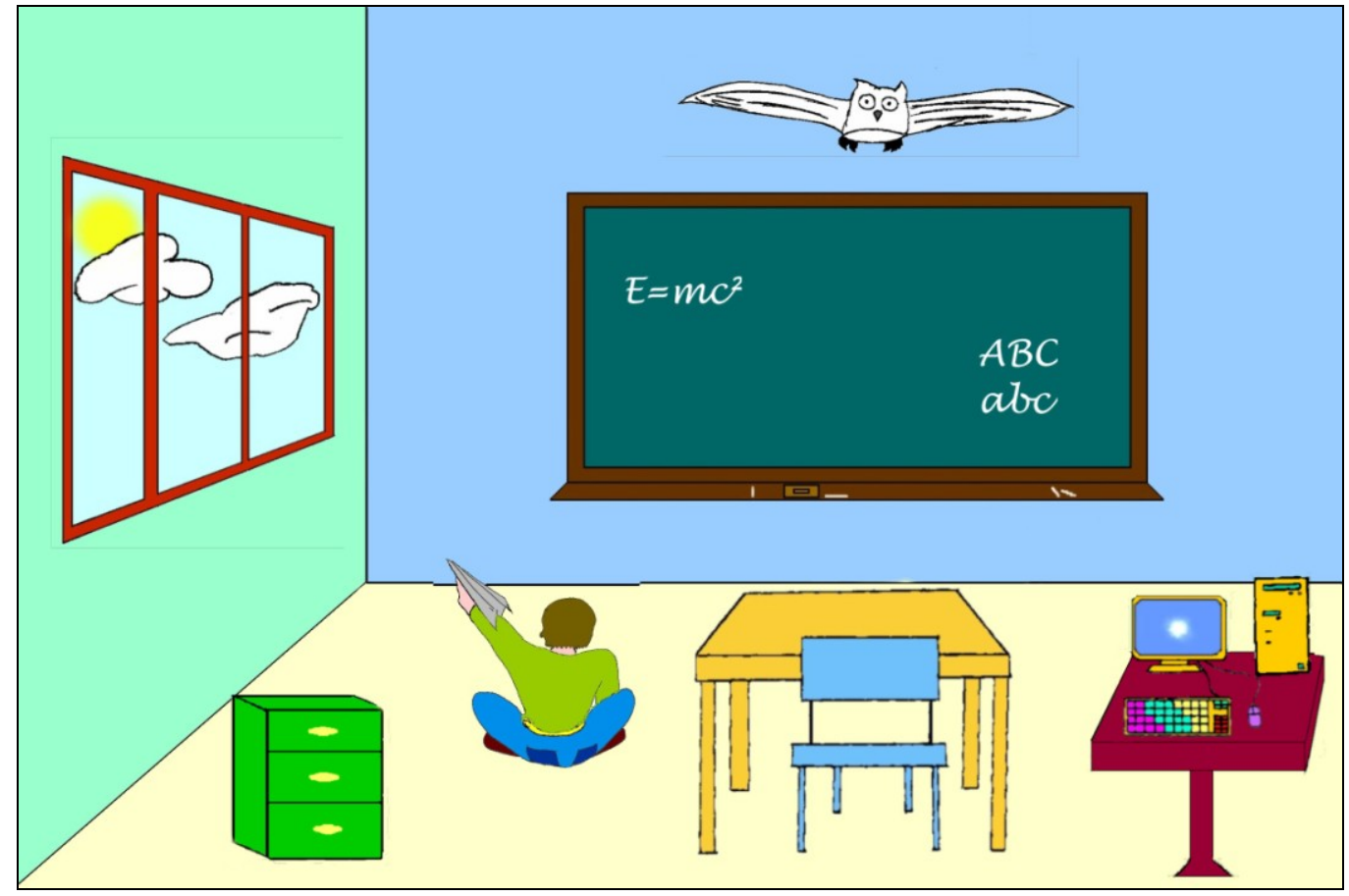

En la elaboración y presentación de las unidades se han tenido en cuenta diferentes estrategias de enseñanza que intentan que el alumnado comprenda mejor la información y pueda asimilarla, este tipo de estrategias se insertan en lo que denomina Hernández (1991) 
estrategias de comunicabilidad didáctica, tales como reducir la densidad cognitiva (poca información en cada pantalla, división de cada unidad en subapartados), estructuración de la información (aparece un esquema de la unidad a la izquierda de la pantalla que el estudiante tiene presente siempre), conexión con los esquemas previos de los estudiantes (uso de ejemplos, analogías), elaboración y producción de la información (actividades abiertas, donde el alumnado debe relacionar y elaborar personalmente la información, enlaces diversos para el acceso rápido a mayor información, etc.). También se han tenido en cuenta estrategias motivacionales en la enseñanza (Hernández, 1991), tales como captar la atención (cambio de colores, fondos diferentes y relativos a cada unidad temática, estímulos visuales, etc.), partir las necesidades o intereses del alumnado (elección de temas a investigar, cuestionarios previos de opinión), generar nuevas necesidades (utilización de preguntas y dilemas en la redacción del texto) y evitar la frustración y generar la probabilidad de éxito en el aprendizaje (guiar el proceso y recibir feedback y refuerzo por parte de los tutores/as). En el gráfico número 3 se presenta un ejemplo de la presentación de una de las unidades, en concreto la sexta, estrategias de personalización.

\section{Gráfica 3: Menú de entrada al programa}

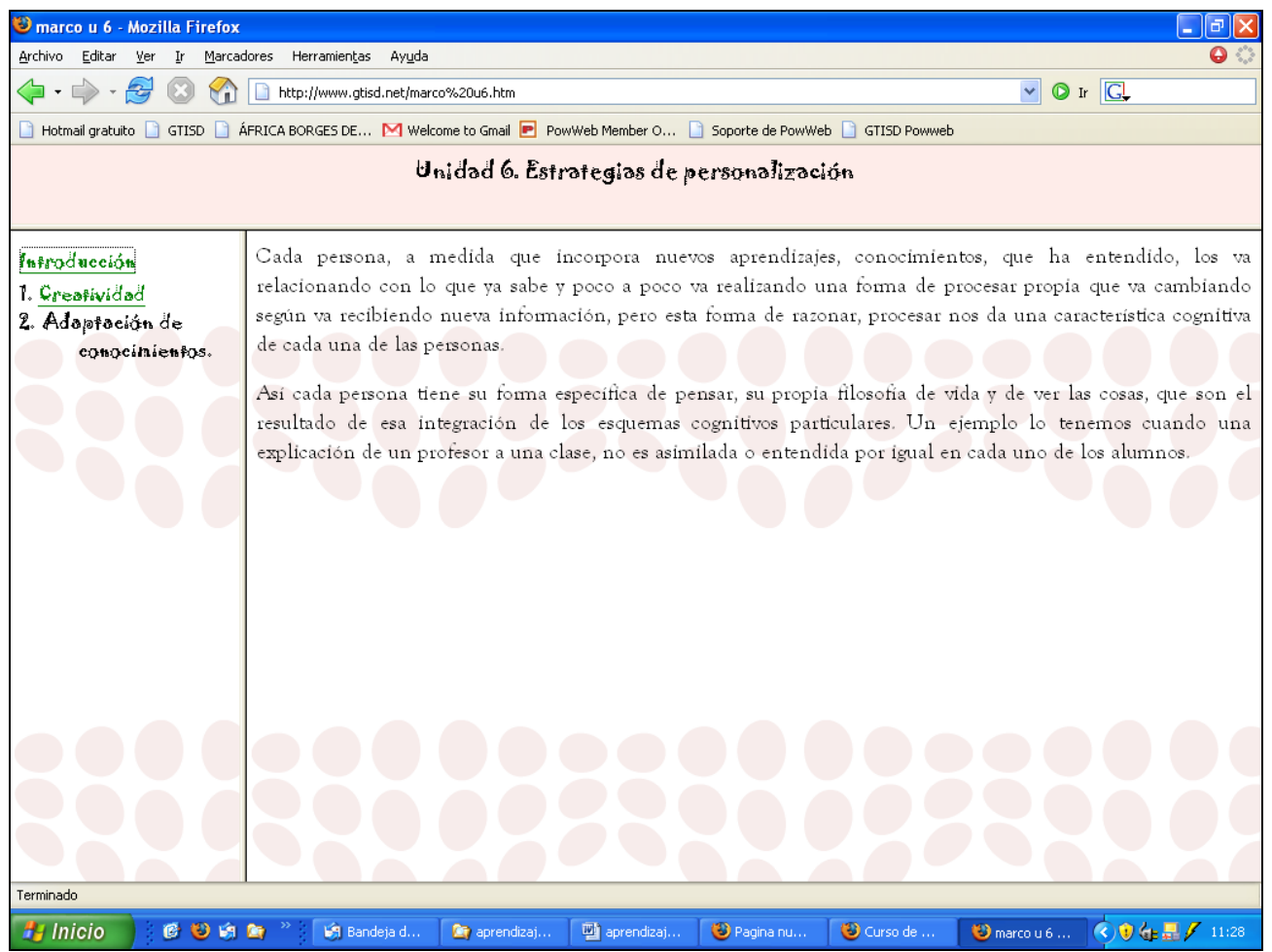

Las actividades que se solicitan al alumnado desde este programa formativo son actividades que se recogen en los fines productivos de la enseñanza (Hernández, 1991), que 
inciden en aspectos motivacionales del alumnado, en el uso de estrategias de aprendizaje y tratan de las siguientes cuestiones:

a) Partir de la experiencia, opiniones, o conocimientos previos de los estudiantes, tales como los cuestionarios que se plantean al inicio de cada una de las unidades.

b) Actividades de elaboración de la información, partiendo de la información previa: redacciones sobre lo que les ha sugerido el tema, reflexión sobre su forma de estudiar, planteamiento de metas, comparaciones sobre la experiencia previa y la actual.

c) Actividades para personalizar el aprendizaje y partir de sus intereses: la elección del tema a investigar, reflexionar sobre su propia manera de estudiar, establecimiento de metas de mejora.

d) Actividades que generan probabilidad de éxito, que van de lo más sencillo a lo complejo: como la búsqueda bibliográfica guiada hasta la búsqueda bibliográfica libre o la guía en el trabajo de elaboración para evitar que el estudiante se siente 'perdido' en el proceso.

e) Actividades que ayudan a afianzar el aprendizaje: cuestionarios sobre el contenido.

f) Actividades metacognitivas: ayudar a planificar el proceso, ayudar y orientar a reflexionar sobre el proceso y planteamiento de metas futuras.

\section{Discusión}

El programa Navegando en el aprendizaje pensamos que puede responder a los objetivos previstos: prevenir posibles problemas de aprendizaje en niños y niñas con altas capacidades, al enseñarle a mejorar sus estrategias de estudio y asimilación de actividades académicas, potenciando el entrenamiento en aprendizaje autorregulado.

No obstante, no es una tarea fácil. Uno de los problemas que hemos observado en el grupo de niños y niñas que hemos tratado en los dos últimos años, es que se muestran muy interesados por todo lo nuevo (Hernández-Jorge, Borges, Rodríguez y García (2004), pero en cambio tienen mayor problema de perseverar en la tarea, a no ser que se les consiga motivar adecuadamente. Por lo tanto, la función del tutor o la tutora resulta especialmente importante, de tal manera que no parezcan lecciones lejanas, al estilo de lo que ya conocen en el aula, sino que se les consiga involucrar, potenciando las ventajas que supone manejar las claves del éxito escolar. 
Ahora queda por comprobar si los datos nos dan la razón. El programa se ha iniciado en octubre de 2004, contando con seis niños y niñas, en edades comprendidas entre los 9 y los 17 años. Esta disparidad de edades, y por lo tanto de formación y de intereses, ha intentado paliarse por la actuación diferencial de cada tutor, personalizando las tareas para su tutorizado. Una de las actividades que abordaremos en breve es elaborar otro nivel inferior del curso, que abarque edades comprendidas entre los 8 y los 11 años, manteniendo este programa para el alumnado de 12 años en adelante. 


\section{Referencias}

Adell, J. (1997). Tendencias en educación en la sociedad de las tecnologías de la información Edutec. Revista electrónica de tecnología educativa, 7, noviembre.

Alcantud, F. (1999). Teleformación: diseño para todos. Valencia: Servicio de publicaciones de la Universidad de Valencia.

Azevedo, R. y Cromley, J. (2004). Does Training on Self-Regulated Learning Facilitate Students'Learning With Hypermedia? Journal of Educational Psychology, 96, 523-535.

Bastani, N. (2004). Fostering Creativity and influencing learning strategies using technology in gifted education. Comunicación presentada en la $9^{\text {th }}$ conference of the European Council for High Ability. Navarra, Septiembre.

Bernardo, J. (2004). Estrategias de aprendizaje. Para aprender más y mejor. Madrid: Rialp.

Bertschi, J. (2004, Septiembre). E-learning and the gifted: studies, comparisons and results from Talenta Zurich. Comunicación presentada en la $9^{\text {th }}$ Conference of the European Council for High Ability. Navarra.

Braggett, E.J., Ashman, A. y Noble, J. (1983). The expressed needs of parents of gifted children. Gifted Education International, 1, 80-83.

Burke, Y. y Tolan, T. (2004, Septiembre). Early chilhood a science/ICT projecft for 6 yerar olds. Comunicación presentada en la $9^{\text {th }}$ Conference of the European Council for High Ability. Navarra.

Castor, S.E. (1997). Locus of control and self-concept in achieving and underachieving gifted students. Dissertation Abstract International, 57(10-B), 6603.

Coltman, T., Devinney, T.M., Latukefu, A.S. y Midgley, D.F. (2002). Keeping e-business in perspective. Communications of the ACM, 45 (8), 69-73

Covington, M.V. (1992). Making the grade: A Slef-Worth perspective on motivation and school form. New York:Cambridge

Cremades, R. (2001). Aprender a estudiar. Claves para mejorar la eficacia en el estudio. Málaga: Arguval.

Flint, L.J. (2003). Self-intervention of gifted underachievers: Stories of success. Dissertation Abstract International, 63(8-a), 2790.

De la Fuente, J. y Martínez, J.M. (2000). Pro\&Regula: Un programa para aprender a regularse durante el aprendizaje (1). Málaga: Ediciones Aljibe. 
García, L.A. (1998). Psicología instruccional e intervención para la mejora cognitiva. Memoria de Cátedra. Universidad de La Laguna.

García, L.A.; Torbay, A.; Rodríguez, N, Martín, E.; Rodríguez, T, y Hernández-Jorge, C. (2003). Ingeniería Mental: Intervención para la mejora de la inteligencia. S/C de Tenerife: Arte Comunicación visual, S.L.

González, R.; Valle, A.; Suárez, J.M. y Fernández, A (2000). Diferencias en los componentes cognitivo y afectivo-motivacional entre distintos niveles de aprendizaje autorregulado en estudiantes universitarios. Bordon, 53 (4).

Hacker, D.J., Dunlonsky, J. y Graesser, A.C. (1998). Metacognition in educational theory and practice. Mahwah, NJ: Lawrence Erlbaum Associates

Hartnett, D.N., Nelson, J.M. y Rinn, A. (2004). Gifted or ADHD? Roeper Review, 26, 73-76.

Hernández, P. (1991). Psicología de la educación: corrientes actuales y teorías aplicadas. México: Trillas.

Hernández, P. y García, L. A. (1991). Psicología y enseñanza del estudio. Madrid: Pirámide.

Hernández-Jorge, C., Borges, A., Rodríguez, M.E. y García, A. (200, Septiembre). Helping teachers cope with gifted child: How teachers feel and react teaching a gifted child in his or hers classroom. Comunicación presentada en la 9th Conference of the European Council for High Ability. Navarra.

Herrera, F.; Ramírez, M.I.; Roa, J.M. y Herrera, I. (2004). ¿Cómo interactúan el autoconcepto y el rendimiento académico en un contexto pluricultural? Revista Iberoamericana de Educación, 34. http://www.campus-oei.org/investigacion6.htm

Jessie, E.; Moore, P.J. y Atputhasamy, L. (2003). High-achieving students: their motivational goals, self-regulation and achievement and relacionships to their teacher's goal and strategy-based instruction. High Ability Studies, 14 (1) 23-39

Kaufmann, F., Kalbfleisch, M.L. y Castellanos, F.X. (2000). Attention deficit disorders and gifted students: What do we really now? (RM00146) Storrs, CT: The National Research Center on the Gifted and Talented, University of Connecticut.

Kunkel, M. A., Chapa, B., Patterson, G., y Walling, D. D. (1992). The experience of giftedness: "Eight great gripes" six years later. Roeper Review, 15, 10-14.

Lee, K. (2001). Using tellecolaboration for Self-regulated Thinking Skills: instruction with regular and gifted learners. High Ability Studies, 12 (2) 235-247

Lee-Corbin, H. y Denicolo, P. (1998). Portraits of the able child: Highlights of case study research. Roeper Review, 9(2), 207-218. 
Marcelo, C. y Lavié, J.M. (2000). Formación y Nuevas Tecnologías: Posibilidades y condiciones de la teleformación como espacio de aprendizaje. Bordón, 52 (3), 385-406.

McCombs, B.L. y Marzano, R.J. (1990). Putting the Self in Self-Regulated Learning: The Self as Agent in Integrating Will and Skill. Educational Psychologist, 25 (1), 51-69.

Neber, H. y Schommer-Aikins, M. (2002). Self-Regulated Science Learning with Highly Gifted Students: the role of cognitive, motivational, epistemological, and environmental variables. High Ability Studies, 13 (1) 59-74

Paris, S.G. y Newman, R.S. (1990). Developmental aspects of self-regulated learning. Educational Psychologist, 25, 87-102.

Pérez, E.; Rubio, C. y Rubio, F. (1999, Septiembre): Modelo de enseñanza-aprendizaje basado en tecnologías de la información. Comunicación presentada en el I Simposium iberoamericano de didáctica universitaria. Santiago de Compostela

Perry, N.; Nordby, C.J. y Vandekamp, K.O. (2003). Promoting self-regulated reading and writing at home and school. The Elementary School Journal, 103 (4), 317-338.

Pintrich, P.R. y Schunk, D.K. (2002). Motivation in Education: Theory, research and implications. Upper Saddle River, NJ: Merrill Prentice-Hall.

Reis, S.M. y McCoach, D.B. (2000). The underachievement of gifted students: What do we know and where we go? Gifted Child Quarterly, 44, 152-170

Reis, S.M. y Colbert, R. (2004). Counseling needs of academically talented students with learning disabilities. Professional School Counseling, 8(2), 156-167.

Rogers, D. y Shawn, K. (2004). Self-regulated Learning and Internet Searching. Teachers College Record, 106, 1084-1105.

Rubio, F. (2000). La influencia de la Tecnología de la información en la Formación. Ponencia impartida en el Curso Superior de Nuevas Tecnologías de la Información y Comunicaciones Aplicadas a la Educación, Formación y Empleo. Universidad de La Laguna.

Ryan, R.M. y Deci, E.L. (2000). Intrinsic and Extrinsic motivations: classic definitions and new directions. Contemporary educational psychology, 25, 54-67.

Shultz, R.A. (2003). Insights from gifted adolescents: Implications for quality gifted education. Documento disponible en Internet: Insigh G- adolescent.pdf (objet aplication/pdf).

Stenberg. R. (1988). The triarchic mind: a new theory of human intelligence. New York, NY: Viking. 
Torbay, A. (2002). Psicología instruccional y mejora de las habilidades cognitivas. Memoria de titularidad. Universidad de La Laguna.

Torbay, A.; Martín, E.; García, L. y Rodríguez, N. (2001 a Septiembre) ¿En qué se diferencian los estudiantes con un estilo creativo de los que no lo tienen en la universidad? Comunicación presentada en el I congreso de creatividad y sociedad. Barcelona.

Torbay, A.; Martín, E.; García, L. y Rodríguez, N. (2001b Septiembre) Estilo creativo y estrategias de aprendizaje en estudiantes universitarios. Comunicación presentada en el I congreso de creatividad y sociedad. Barcelona.

Weinfeld, R., Barnes-Robinson, L., Jeweler, S. y Shevitz, B. (2002). Academic programs for gifted and talented/learning disabled students. Roeper Review, 24(4), 226-233.

Wigfield, A. y Eccles, J.S. (2000). Expectancy-value theory of achievement motivation. Contemporary educational psychology, 25, 68-81.

Winne, P.H. y Perry, N.E. (2000). Measuring self-regulated learning. En P.Pintrich, M. Boekaerts y M. Zeidner (Eds). Handbook of self- regulation (pp532-566). Orlando, FL: Academic Press.

Zimmerman, B.J. (1990). Self-regulated learning and academic achievement: An overview. Educational Psychologist, 25, 3-17.

Zimmerman, B.J. (1995). Self-regulation involves more than metacognitions: a social cognitive perspective. Educational psychologist, 30, 217-221

Zentall, S.S., Hall, A.M. y Grskovic, J.A. (2001). Learning and motivational characteristics of boys with AD/HD and/or giftedness. Exceptional Children, 67, 499-519

Zimmerman, B.J. y Bandura, A. (1994). Impact of self-regulatory influences on writing course attainment. American Educational Research Journal, 31, 845-862.

Zimmerman, B.J. y Martínez-Pons, M. (1990). Student Differences in Self-Regulated Learning: Relating Grade, Sex and Giftedness to Self-Efficacy and Stratefy Use. Journal of educational Psychology, 82 (1) 51-59.

Zimmerman, B.J. y Schunk, D.H. (2001). Reflections on theories of self-regulated learning and academic achievement. En B.J. Zimmerman y D.H. Schunk Self-regulated learning and academic achievement: Theoretical perspectives (2nd ed., págs. 289307. Mahwah, NJ: Lawrence Erlbaum Associates. 


\section{ANEXO}

\section{Contenidos del programa Navegando en el aprendizaje}

\section{Unidad 1: Recogida de información}

1.1.- Utilidad de recoger información

1.2.- El conocimiento científico

1.3.- Estudio e investigación

1.4.-Formas de recogida de información

1.5.-El saber formal: libros y bibliotecas

1.6.- Nuevas formas de acceso a información: Internet

1.7.- Recapitulación

\section{Unidad2: Estrategias de apoyo}

2.1.- Estrategias para condiciones físicas y ambientales

2.2.- Estrategias de condiciones psicológicas:

a) Actitudes de voluntad

b) Actitud personal ante el estudio

c) Condiciones sobre las propias actitudes para aprender

2.3.-Recapitulación

\section{Unidad 3: Estrategias de atención}

3.1.- Captación y selección de la información

3.2.- La atención en el mensaje oral

3.3.- La atención en el lenguaje escrito

Unidad 4: Procesamiento de la información (1): Estrategias para la elaboración y organización de la información.

4.1. Códigos para la representación mental de los conocimientos a aprender

4.2. Mapas conceptuales y esquemas

4.3. Recapitulación

Unidad 5: Procesamiento de la información (2): Estrategias de memorización.

5.1. Tipos y elementos de la memoria 
5.2. Estrategias de memorización

\section{Unidad 6: Estrategias de personalización}

6.1.- La creatividad

6.2.-La transferencia o generalización de los conocimientos integrados

6.3.- Recapitulación

\section{Unidad 7: Difusión de los conocimientos}

7.1. Preparación y rendimiento en los exámenes

7.2.- Elaboración escrita de la información

7.3.- Presentaciones orales

7.4.- Recapitulación final 